\title{
Close Binaries with Two Compact Objects
}

\author{
V. Kalogera \\ Harvard-Smithsonian Center for Astrophysics, 60 Garden St., \\ Cambridge, MA 02138, USA
}

\begin{abstract}
The coalescence of close binary systems with two compact objects (neutron stars and black holes) are considered to be promising sources of gravitational waves for the currently built laser interferometers. Here, I review the current Galactic coalescence estimates derived both theoretically and empirically. I discuss the uncertainties involved as well as ways of obtaining an upper limit to the coalescence rate of two neutron stars.
\end{abstract}

\section{Introduction}

The late inspiral phase due to gravitational radiation of close binaries with two compact objects, neutron stars (NS) or black holes (BH), is expected to be a major source of gravitational waves for ground-based interferometers, such as LIGO and VIRGO). The binary radio pulsar PSR B1913+16 (Hulse \& Taylor 1975 ) is the prototypical NS-NS system and has provided a remarkable empirical confirmation of general relativity with the measurement of orbital decay due to gravitational radiation (Taylor \& Weisberg 1982). In addition to NS-NS binaries, the existence of close $\mathrm{BH}-\mathrm{NS}$ and $\mathrm{BH}-\mathrm{BH}$ systems has also been predicted theoretically, and they are considered as possible sources of gravitational radiation.

The expected detection rate of inspiral events depends crucially on the rate of such events in the Galaxy and by extrapolation out to the maximum distance an inspiral event could be detected. In what follows I review the current estimates of Galactic coalescence rates, obtained theoretically and empirically, and I discuss the associated uncertainties. For the case of NS-NS binaries, I also discuss a new way of obtaining an empirical upper bound to their coalescence rate. The scale for all these rates is set by requiring that the detection rate for the "enhanced" LIGO is $\sim 2-3$ events per year. Given the amplitude of the signals (e.g., Thorne 1996), the requirement for the NS-NS Galactic rate is $\sim 10^{-5} \mathrm{yr}^{-1}$ (detected out to $\sim 200 \mathrm{kpc}$ ) and for the BH-BH Galactic rate $\sim 2 \times 10^{-7} \mathrm{yr}^{-1}$ (detected out to $\sim 700 \mathrm{kpc}$ ).

\section{Theoretical Estimates}

The formation rate of coalescing binary compact objects (i.e., systems in close enough orbits that coalesce within a Hubble time) can be calculated, given a sequence of evolutionary stages leading to binary compact object formation. Over 
the years a relatively standard picture (van den Heuvel 1976) has been formed describing the birth of such systems based on the consideration of NS-NS binaries (for variations of this picture see Brown 1995; Terman \& Taam 1995): The initial binary progenitor consists of two binary members massive enough to eventually collapse into a NS or a BH. The evolutionary path involves multiple phases of stable or unstable mass transfer, common-envelope phases, and accretion onto compact objects, as well as two core collapse events. Theoretical modeling of this formation process has been undertaken by various authors using population synthesis techniques, Such studies provide us with $a b$ initio predictions of coalescence rates. The evolution of an ensemble of primordial binaries with assumed initial properties is followed until a coalescing binary is formed. The changes in the binary properties at the end of each evolutionary stage are calculated based on our current understanding of the processes involved: wind mass loss from massive hydrogen- and helium-rich stars, mass and angular-momentum losses during mass transfer phases, dynamically unstable mass transfer and commonenvelope evolution, effects of highly super-Eddington accretion onto NS, and supernova explosions with kicks imparted to newborn NS or even BH. Given that several of these phases are not well understood, the results of population synthesis are expected to depend on the assumptions made in the treatment of the various processes. Therefore, exhaustive parameter studies are required by the nature of the problem.

Recent studies of the formation rate of coalescing compact objects (Lipunov, Postnov, \& Prokhorov 1997; Fryer, Burrows, \& Benz 1998; Portegies-Zwart \& Yungel'son 1998; Brown \& Bethe 1998; Fryer, Woosley, \& Hartmann 1999) have explored the input parameter space and the robustness of the results at different levels of (in)completeness. Kicks imparted to NS and BH at birth are the best studied of all model assumptions. In the case of NS-NS coalescence the range of predicted Galactic rates is $<10^{-7}-5 \times 10^{-4} \mathrm{yr}^{-1}$. This large range indicates the importance of supernovae (two in this case) in binaries. Variations in the assumed mass-ratio distribution for the primordial binaries can further change the predicted rate by about a factor of 10, while assumptions of the common-envelope phase add another factor of about $10-100$. Variation in other parameters typically affects the results by factors of two or less. Predicted rates for $\mathrm{BH}-\mathrm{NS}$ and $\mathrm{BH}-\mathrm{BH}$ binaries lie in the ranges $<10^{-7}-10^{-4} \mathrm{yr}^{-1}$ and $<10^{-7}-10^{-5} \mathrm{yr}^{-1}$, respectively when the kick magnitude to both $\mathrm{NS}$ and $\mathrm{BH}$ is varied. Other uncertain factors such as the critical progenitor mass for NS and $\mathrm{BH}$ formation lead to variations of the rates by factors of $10-50$.

Although results from population syntheses regarding binary properties, such as orbital sizes, eccentricities, center-of-mass velocities, can be quite robust, it is evident that predicted rates, i.e., the absolute normalization of the models, cover a wide range of values (typically $3-4$ orders of magnitude). Given these results it seems fair to say that population synthesis calculations have a rather limited predictive power and provide fairly loose constraints on coalescence rates.

\section{Empirical Estimates}

The observed sample of coalescing NS-NS binaries (PSR B1913+16 and PSR B1534+12) provides us with another way of estimating their coalescence rate. 
An empirical estimate can be obtained using their observed pulsar and binary properties along with models of selection effects in radio pulsar surveys. For each observed object, a scale factor can be calculated based on the fraction of the Galactic volume within which pulsars with properties identical to those of the observed pulsar could be detected by any of the radio pulsar surveys, given their detection thresholds. This scale factor is a measure of how many more pulsars like the ones detected in the coalescing NS-NS systems exist in our galaxy. The coalescence rate can then be calculated based on the scale factors and estimates of detection lifetimes summed up for all the observed systems. This basic method was first used by Phinney (1991) and Narayan, Piran, \& Shemi (1991) who estimated the Galactic rate to be $\sim 10^{-6} \mathrm{yr}^{-1}$.

Since then, estimates of the NS-NS coalescence rate have known a significant downward revision primarily because of (i) the increase of the Galactic volume covered by radio pulsar surveys with no additional coalescing NS-NS being discovered (Curran \& Lorimer 1995), (ii) the increase of the distance estimate for PSR B1534+12 based on measurements of post-Newtonian parameters (Stairs et al. 1998) (iii) revisions of the lifetime estimates (van den Heuvel \& Lorimer 1996; Arzoumanian, Cordes, \& Wasserman 1999). In addition, a significant upward correction factor $(\sim 7-10)$ has been used recently to account for the faint end of the radio pulsar luminosity function. The most recently published study (Arzoumanian et al. 1999) gives a lower limit of $2 \times 10^{-7} \mathrm{yr}^{-1}$ and a "best" estimate of $\sim 6-10 \times 10^{-7} \mathrm{yr}^{-1}$, which agrees with other recent estimates of $2-3 \times 10^{-6} \mathrm{yr}^{-1}$ (Stairs et al. 1998; Evans et al. 1999). Additional uncertainties arise from estimates of pulsar ages and distances, the pulsar beaming fraction, the spatial distribution of DNS in the Galaxy.

Despite all these uncertainties the empirical estimates of the NS-NS coalescence rate appear to span a range of $\sim 1-2$ orders of magnitude, which is narrow compared to the range covered by the theoretical estimates.

\subsection{Small Number Sample and Pulsar Luminosity Function}

One important limitation of empirical estimates of the coalescence rates is that they are derived based on only two observed NS-NS systems, under the assumption that the observed sample is representative of the true population, particularly in terms of their radio luminosity. Assuming that DNS pulsars follow the radio luminosity function of young pulsars and that therefore their true population is dominated in number by low-luminosity pulsars, it can be shown that the current empirical estimates most probably underestimate the true coalescence rate. If a small-number sample is drawn from a parent population dominated by low-luminosity (hence hard to detect) objects, it is statistically more probable that the sample will actually be dominated by objects from the high-luminosity end of the population. Consequently, the empirical estimates based on such a sample will tend to overestimate the detection volume for each observed system, and therefore underestimate the scale factors and the resulting coalescence rate.

This effect can be clearly demonstrated with a Monte Carlo experiment (Kalogera et al. 1999) using simple models for the pulsar luminosity function and the survey selection effects. As a first step, the average observed number of pulsars is calculated given a known "true" total number of pulsars in the Galaxy (thick-solid line in Figure 1a). As a second step, a large number of 

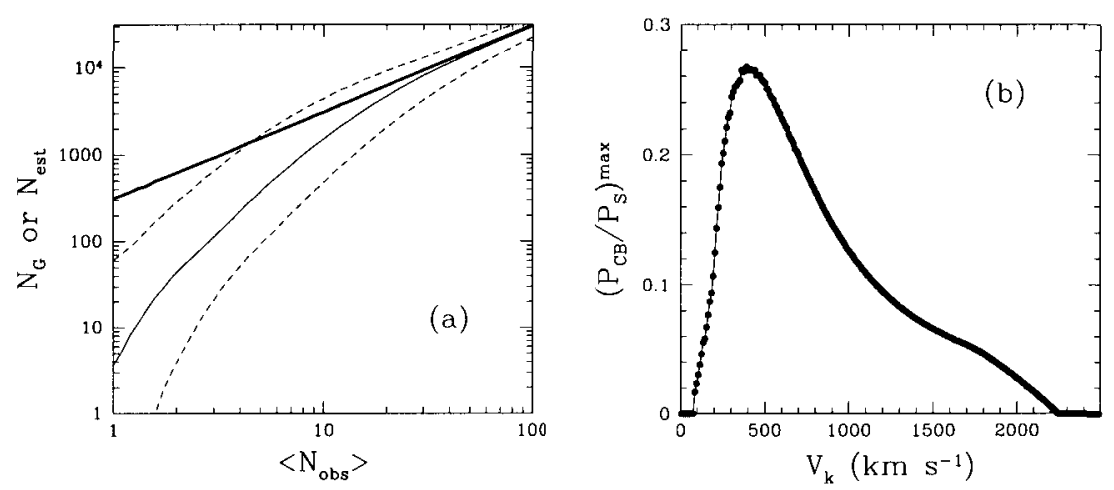

Figure 1. (a) Bias of the empirical estimates of the DNS coalescence rate because of the small-number observed sample. See text for details. (b) Maximum probability ratio for the formation of coalescing DNS and the disruption of binaries as a function of the kick magnitude at the second supernova.

sets consisting of "observed" (simulated) pulsars are realized using Monte Carlo methods. These pulsars are drawn from a Poisson distribution of a given mean number $\left(<N_{\text {obs }}\right\rangle$ ) and have luminosities assigned according to the assumed luminosity function. Based on each of these sets, one can estimate the total number of pulsars in the Galaxy using empirical scale factors, as is done for the real observed sample. The many (simulated) 'observed' samples can then be used to obtain the distribution of the estimated total Galactic numbers $\left(N_{\text {est }}\right)$ of pulsars. The median and $25 \%$ and $75 \%$ percentiles of this distribution are plotted as a function of the assumed number of systems in the (fake) 'observed' samples in Figure 1a (thin-solid and dashed lines, respectively).

It is evident that, in the case of small-number observed samples (less than $\sim 10$ objects), the estimated total number, and hence the estimated coalescence rate, can be underestimated by a significant factor. For a two-object sample, for example, the true rate maybe higher by more than a factor of ten. This correction factor associated with the faint-end of the luminosity function can be applied to the estimated NS-NS coalescence rate in place of the factor of $\sim 7-10$ used so far from a direct extrapolation of the luminosity function.

\section{Limits on the NS-NS Coalescence Rate}

Observations of NS-NS systems and isolated pulsars related to NS-NS formation allow us to obtain upper limits on their coalescence rate. Depending on how their value compares to the "enhanced" LIGO requirement such limits can provide us with valuable information about the prospects of gravitational-wave detection.

Bailes (1996) used the absence of any young pulsars detected in NS-NS systems and obtained a rough upper limit to the rate of $\sim 10^{-5} \mathrm{yr}^{-1}$, while 
recently Arzoumanian et al. (1999) reexamined this in more detail and claimed a more robust upper limit of $\sim 10^{-4} \mathrm{yr}^{-1}$.

An upper bound to the NS-NS coalescence rate can also be obtained by combining our theoretical understanding of orbital dynamics (for supernovae with NS kicks in binaries) with empirical estimates of the birth rates of other types of pulsars related to NS-NS formation (Kalogera \& Lorimer 2000, hereafter KL00). Progenitors of NS-NS systems experience two supernova explosions. The second supernova explosion (forming the NS that is not observed as a pulsar) provides a unique tool for the study of NS-NS formation, since the post-supernova evolution of the system is simple, driven only by gravitationalwave radiation. There are three possible outcomes after the second supernova: (i) a coalescing NS-NS is formed (CB), (ii) a wide NS-NS (with a coalescence time longer than the Hubble time) is formed (WB), or (iii) the binary is disrupted (D) and a single pulsar similar to the ones seen in NS-NS systems is ejected. Based on supernova orbital dynamics we can accurately calculate the probability branching ratios for these three outcomes, $P_{\mathrm{CB}}, P_{\mathrm{WB}}$, and $P_{\mathrm{D}}$. For a given kick magnitude, we can calculate the maximum ratio $\left(P_{\mathrm{CB}} / P_{\mathrm{D}}\right)^{\text {max }}$ for the complete range of pre-supernova parameters defined by the necessary constraint $P_{\mathrm{CB}} \neq 0$ (Figure 1b). Given that the two types of systems have a common parent progenitor population, the ratio of probabilities is equal to the ratio of the birth rates $\left(B R_{\mathrm{CB}} / B R_{\mathrm{D}}\right)$.

We can then use $(i)$ the absolute maximum of the probability ratio $(\approx 0.26$ from Figure 1b) and (ii) an empirical estimate of the birth rate of single pulsars similar to those in NS-NS based on the current observed sample to obtain an upper limit to the coalescence rate. The selection of this sample involves some subtleties (KL00), and the analysis results in $B R_{\mathrm{CB}}<1.5 \times 10^{-5} \mathrm{yr}^{-1}$ (KL00). Note that this number could be increased because of the small-number sample and luminosity bias affecting this time the empirical estimate of $B R_{\mathrm{D}}$ by a factor of 2-6 (Kalogera et al. 1999).

This is an example of how we can use observed systems other than NS-NS to improve our understanding of their coalescence rate. A similar calculation can be done using the wide NS-NS systems instead of the single pulsars (see KLO0).

\section{Conclusions}

The current theoretical estimates of NS-NS coalescence rates appear to have a rather limited predictive power. They cover a range in excess of 3 orders of magnitude and most importantly this range includes the value of $10^{-5} \mathrm{yr}^{-1}$ required for an "enhanced" LIGO detection rate of 2-3 events per year. This means that at the two edges of the range the conclusion swings from no detection to many per month, and therefore the detection prospects of NS-NS coalescence cannot be assessed firmly. On the other hand empirical estimates derived based on the observed sample appear to be more robust (estimates are all within a factor smaller than 100). Given these we would expect a detection of one event every few years up to ten events per year.

Estimates of the coalescence rate of $\mathrm{BH}-\mathrm{NS}$ and $\mathrm{BH}-\mathrm{BH}$ systems rely solely on our theoretical understanding of their formation. As in the case of NS-NS 
binaries, the model uncertainties are significant and the ranges extend to more than 2 orders of magnitude. However, the requirement on the Galactic rate is less stringent for $10 \mathrm{M}_{\odot} \mathrm{BH}-\mathrm{BH}$ binaries, only $\sim 2 \times 10^{-7} \mathrm{yr}^{-1}$. Therefore, even with the pessimistic estimates for $\mathrm{BH}-\mathrm{BH}$ coalescence rates $\left(\sim 10^{-7} \mathrm{yr}^{-1}\right)$, we would expect at least a few detections per year, which is quite encouraging. We note that a very recent examination of dynamical $\mathrm{BH}-\mathrm{BH}$ formation in globular clusters (Portegies-Zwart \& McMillan 1999) leads to detection rates as high as a few per day.

Acknowledgments. I would like to thank the organizers of the meeting for their invitation and support of my participation. I also acknowledge support by the Smithsonian Institute in the form of a CfA Post-doctoral Fellowship.

\section{References}

Arzoumanian, Z., Cordes, J.M., \& Wasserman, I. 1999, ApJ, 520, 696

Bailes, M. 1996, in IAU Symp. 165, Compact Stars in Binaries, ed. J. van Paradijs, E. P. J. van den Heuvel \& E. Kuulkers (Kluwer Academic Publishers, Dordrecht), 213

Brown, G.E. 1995, ApJ, 440, 270

Brown, G.E. \& Bethe, H. 1998, ApJ, 506, 780

Curran, S.J. \& Lorimer, D.R. 1995, MNRAS, 276, 347

Evans, T., et al. 1999, to appear in the proceedings of the XXXIVth Rencontres de Moriond on Gravitational Waves and Experimental Gravity, Les Arcs, France.

Fryer, C.L., Burrows, A., Benz, W. 1998, ApJ, 496, 333

Fryer, C.L., Woosley, S.E., Hartmann, D. 1999, ApJ, in press [astro-ph/9904122]. Hulse, R.A., \& Taylor, J.H. 1975, ApJ, 195, L51

Kalogera, V. \& Lorimer, D.R. 2000, ApJ, 530, in press [astro-ph/9907426].

Kalogera, V., Narayan, R., Spergel, D., \& Taylor, J.H. 1999, ApJ, in preparation Lipunov, V.M., Postnov, K.A., Prokhorov, M.E. 1997, MNRAS, 288, 245

Narayan, R., Piran, T., \& Shemi, A. 1991, ApJ, 379, L17

Phinney, E.S. 1991, ApJ, 380, L17

Portegies-Zwart, S.F. \& McMillan, S.L.W., ApJ, submitted [astro-ph/9910061].

Portegies-Zwart, S.F. \& Yungel'son, L.R. 1998, A\&A, 332, 173

Stairs, I.H., et al. 1998, ApJ, 505, 352

Terman, J.L., \& Taam, R.E. 1995, MNRAS, 276, 1320

Thorne, K.S. 1996, in IAU Symp. 165, Compact Stars in Binaries, ed. J. van Paradijs, E. P. J. van den Heuvel \& E. Kuulkers (Kluwer Academic Publishers, Dordrecht), 153

van den Heuvel, E.P.J. 1976, in IAU Symp. 73, Structure and Evolution of Close Binary Systems, ed. P. Eggleton, S. Mitton \& J. Whelan (Kluwer Academic Publishers, Dordrecht).

van den Heuvel, E.P.J. \& Lorimer, D.R. 1996, MNRAS, 283, 37 